Original Research

\title{
Preclinical and clinical evidence of activity of pazopanib in solitary fibrous tumour
}

\author{
S. Stacchiotti ${ }^{\text {a,* }}$, M. Tortoreto ${ }^{\text {b }}$, G.G. Baldi ${ }^{\mathrm{c}}$, G. Grignani ${ }^{\mathrm{d}}$, A. Toss ${ }^{\mathrm{e}}$, \\ G. Badalamenti ${ }^{\mathrm{f}}$, D. Cominetti ${ }^{\mathrm{b}}$, C. Morosi ${ }^{\mathrm{g}}$, A.P. Dei Tos ${ }^{\mathrm{h}}$, F. Festinese ${ }^{\mathrm{i}}$, \\ E. Fumagalli ${ }^{\mathrm{a}}$, S. Provenzano ${ }^{\mathrm{a}}$, A. Gronchi ${ }^{\mathrm{j}}$, E. Pennacchioli ${ }^{\mathrm{k}}$, T. Negri ${ }^{1}$, \\ G.P. Dagrada ${ }^{1}$, R.D. Spagnuolo ${ }^{1}$, S. Pilotti ${ }^{1,1}$, P.G. Casali ${ }^{\mathrm{a}, 1}$, N. Zaffaroni ${ }^{\mathrm{b}}$
}

\footnotetext{
a Adult Mesenchymal Tumor Medical Oncology Unit, Cancer Medicine Department, Fondazione IRCCS Istituto Nazionale Tumori, Milan, Italy

${ }^{\mathrm{b}}$ Molecular Pharmacology Unit, Department of Experimental Oncology and Molecular Medicine, Fondazione IRCCS Istituto Nazionale

Tumori, Milan, Italy

' Medical Oncology Unit 'Sandro Pitigliani', S. Stefano Civil Hospital, Prato, Italy

${ }^{\mathrm{d}}$ Medical Oncology, IRCCS - Istituto di Candiolo, Candiolo, Italy

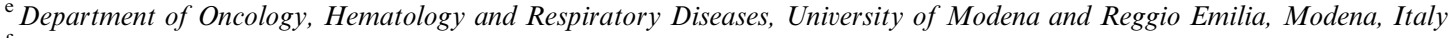

${ }^{\mathrm{f}}$ Department of Oncology, University Hospital of Palermo, Palermo, Italy

${ }^{\mathrm{g}}$ Department of Radiology, Fondazione IRCCS Istituto Nazionale Tumori, Milan, Italy

${ }^{\mathrm{h}}$ Department of Anatomic Pathology, General Hospital of Treviso, Treviso, Italy

${ }^{\mathrm{i}}$ Pharmacy Unit, Fondazione IRCCS Istituto Nazionale Tumori, Milan, Italy

${ }^{\mathrm{j}}$ Melanoma and Sarcoma Unit, Department of Surgery, Fondazione IRCCS Istituto Nazionale Tumori, Milan, Italy

${ }^{\mathrm{k}}$ Melanoma and Sarcoma, Surgery Department, Istituto Europeo di Oncologia, Milan, Italy

${ }^{1}$ Laboratory of Experimental Molecular Pathology, Department of Diagnostic Pathology and Laboratory, Fondazione IRCCS Istituto Nazionale Tumori, Milan, Italy
}

Received 5 June 2014; received in revised form 10 August 2014; accepted 8 September 2014 Available online 27 September 2014

\section{KEYWORDS}

Sarcoma

Solitary fibrous tumour

Pazopanib

Sunitinib

Tyrosine kinase

Chemotherapy

\begin{abstract}
Background: To explore the activity of pazopanib in solitary fibrous tumour (SFT). Patients and methods: In a preclinical study, we compared the activity of pazopanib, sorafenib, sunitinib, regorafenib, axitinib and bevacizumab in a dedifferentiated-SFT (DSFT) xenotransplanted into Severe Combined Immunodeficiency (SCID) mice. Antiangiogenics were administered at their reported optimal doses when mean tumour volume (TV) was $80 \mathrm{~mm}^{3}$. Drug activity was assessed as TV inhibition percentage (TVI\%). From May 2012, six consecutive patients with advanced SFT received pazopanib, on a national name-based programme. In one case sunitinib was administered after pazopanib failure.
\end{abstract}

\footnotetext{
* Corresponding author at: Fondazione IRCCS Istituto Nazionale dei Tumori, via Venezian 1, Milano, Italy. Tel.: +39 0223902803; fax: +39 0223902404.

E-mail address: silvia.stacchiotti@istitutotumori.mi.it (S. Stacchiotti).

${ }^{1}$ These authors equally contributed to the paper.
} 
Results: In the xenograft model, pazopanib showed the lowest antitumour activity (21\%TVI), while regorafenib was the most active $(95 \%$ TVI). Sorafenib, bevacizumab, sunitinib were markedly active (78/70/65\%TVI). Axitinib was marginally active (51\%TVI).

In the retrospective case-series, three patients carried malignant-SFT (MSFT), three DSFT. Best Response Evaluation Criteria in Solid Tumour (RECIST) responses were: three stable disease (SD), all MSFT, three progressive disease (PD), all DSFT, corresponding to one partial response (PR), two SD, three PD by Choi criteria. Median-progression-free survival was 3 months (range 1-15). In one patient, sunitinib was started after pazopanib failure, with a response.

Conclusions: In dedifferentiated-SFT xenograft pazopanib induced a marginal antitumour activity, while regorafenib appeared the most active and promising agent. When administered in patients, pazopanib showed a modest activity in terms of tumour growth stabilisation, observed only in non-dedifferentiated cases.

(c) 2014 Elsevier Ltd. All rights reserved.

\section{Introduction}

Pazopanib is an inhibitor of vascular endothelial growth factor receptor (VEGFR) 1-3 recently approved for treatment of non-adipocytic advanced soft tissue sarcoma (STS) after failure to front-line chemotherapy [1]. In a Phase 3 study on pazopanib in non-adipocytic STS the median progression-free survival (PFS) was 4.6 months for pazopanib compared with 1.6 months for placebo, with an overall survival (OS) of 12.5 months versus 10.7 months [2].

Very few data are available on the activity of pazopanib in solitary fibrous tumour (SFT), a rare STS subtype [3], the sensitivity of which to antiangiogenics like sorafenib, sunitinib and bevacizumab is reported [4-9]. Antiangiogenics were shown to produce durable disease stabilisation in a proportion of patients by means of tumour responses that were mostly non-dimensional $[5,6]$. We already reported on the activity of pazopanib in a human high-grade dedifferentiated-SFT xenotransplanted into severe combined immunodeficiency (SCID) mice [10]. When compared to dacarbazine and temozolomide, pazopanib was less active and characterised by a short lasting cytostatic effect. Thus, we decided to expand that experiment to a broader number of antiangiogenic agents (i.e. sorafenib, regorafenib, axitinib in addition to sunitinib and bevacizumab) already applied in the clinical practice. Our findings are reported herein.

Within a name-based protocol following the results of the Phase 3 trial, we treated with pazopanib six patients affected by SFT. We report herein their outcome as well.

\section{Patients and methods}

\subsection{Experimental model and pharmacological studies}

A patient-derived human high-grade dedifferentiatedSFT xenograft model [10] was used in the study. The presence of the typical NAB2-STAT6 rearrangement - recently described in human SFT [12] - was confirmed in xenograft by RT-PCR [10].

The xenograft model was maintained by serial subcutis (s.c.) passages in 6 week-old female SCID mice (Charles River, Calco, IT). Briefly, when tumours reached approximately $500 \mathrm{~mm}^{3}$, they were removed, aseptically dissected, cut into small fragments $(3 \times 3 \times 3 \mathrm{~mm})$ and s.c. implanted in the mouse right flank. Twenty-four hours after tumour inoculum, $100 \mu \mathrm{L}$ of Matrigel Basement Matrix (BD Biosciences) was injected intratumourally. Mice were housed in a pathogen-free facility with free access to food and water. Tumour growth was followed by biweekly measurement of tumour diameters with a Vernier caliper, and tumour volume (TV) was calculated according to the following formula: TV $\left(\mathrm{mm}^{3}\right)=d^{2} \times D / 2$, where $d$ and $D$ are the shortest and the longest diameter, respectively.

\subsubsection{Xenograft treatment}

Treatment was started when xenotransplanted tumours were approximately $80 \mathrm{~mm}^{3}$ (day 35). Eight mice for each group were used. Pazopanib, sorafenib, sunitinib, regorafenib and axitinib were all dissolved in $0.5 \%$ carboxymethylcellulose and delivered by oral gavage 5 days/week for 4 weeks $(\mathrm{qd} \times 5 \mathrm{~d} / \mathrm{w} \times 4 \mathrm{w}) \times 2$ after a 3 -week rest at their reported optimal dose of 100/60/40/ 30 and $2 \times 25 \mathrm{mg} / \mathrm{kg}$, respectively. Bevacizumab was delivered intraperitoneally twice a week for 4 weeks $(\mathrm{q} 3-4 \mathrm{~d} / \mathrm{w} \times 4 \mathrm{w}) \times 2$ after a 3 -week rest at its reported optimal dose of $4 \mathrm{mg} / \mathrm{kg}$ [12-16]. Control mice were treated with vehicle.

Antitumour activity was assessed as tumour volume inhibition percentage $(\mathrm{TVI} \%)$ in treated versus control mice $(\mathrm{TVI} \%=100-(\mathrm{T} / \mathrm{C} \times 100) \times 100$, where $\mathrm{T}$ and $\mathrm{C}$ are the mean tumour volume of treated and control mice, respectively). Drug toxicity was determined as body weight loss and lethal toxicity.

The use of patient material in xenograft and all the experiments were approved by the Ethics Committee for Animal Experimentation of Fondazione IRCCS 
Istituto nazionale dei tumori Milan, Italy (INT), in compliance with national and international law and policies.

\subsubsection{PDGFRB, VEGFR2 and ERK1/2 expression/ activation}

PDGFRB expression/activation was analysed by Western blotting (WB) on $20 \mu \mathrm{g}$ of protein lysates using anti-PDGFRB (1:1000; \#4564; Cell Signaling Technology, Danvers, MA) and anti-phospho-PDGFRB (1:1000; \#3166 Tyr751; Cell Signaling Technology) antibodies. Anti-actin antibody (1:2500; A2066; Sigma-Aldrich, St. Luis, MO) was used to ensure equal loading of proteins and to normalise the results.

VEGFR 2 was analysed by immunoprecipitation (IP)/ WB: equal amounts $(1 \mathrm{mg})$ of protein lysates were precipitated by incubation with anti-VEGFR2 Sepharose bead Conjugate (\#5168; Cell Signaling Technology). WB was carried-out using anti-phosphotyrosine antibody (1:3000; 05-321; Merck Millipore, Billerica, MA) to detect VEGFR2 activation. The filters were stripped and incubated with anti-VEGFR2 antibody (1:1000; \#2479; Cell Signaling Technology) to evaluate VEGFR2 degree of expression.

ERK $1 / 2$ expression and activation were evaluated by WB on $20 \mu \mathrm{g}$ of protein lysates using anti-phosphoMAPK (1:1000; \#4376 Thr202/Tyr204; Cell Signaling Technology) and anti-MAPK (1:1000; \#9102 p44/42; Cell Signaling Technology) antibodies. Anti-actin antibody was used to ensure equal loading of proteins and to normalise the results.

\subsection{Patients}

We identified six patients with metastatic SFT consecutively treated within the Italian name-based programme on pazopanib in advanced STS, open from May 2012 to May 2013. Patients were treated at INT and in other four Italian institutions. Only cases whose diagnosis was centrally confirmed by expert sarcoma pathologists (SP and APDT) are included in this study. Performance status $(\mathrm{ECOG}) \leqslant 3$, adequate bone marrow and organ function, past medical history negative for deep vein thrombosis, pulmonary embolism or cerebral vascular disorder were requested.

All patients provided a written informed consent to the treatment with pazopanib. Approval by each Institutional Review Board was also required.

\subsubsection{Treatment}

Patients received oral pazopanib $800 \mathrm{mg} /$ day (i.e. $400 \mathrm{mg} \times 2 /$ day), continuously, until progression or toxicity. Treatment was withheld for haematologic grade $(G) \geqslant 3$ and for non-haematologic $G \geqslant 2$ adverse events (AE) as defined according to the National Cancer Institute Common Toxicity Criteria, version 3.0, and restarted after recovery to $\mathrm{G}<2$ in case of haematologic or $\mathrm{G}<1$ in case of non-haematologic.

\subsubsection{Clinical assessment}

Biochemistry and blood count were evaluated at baseline and monitored throughout the study period. $\mathrm{AE}$ were recorded. Disease status was assessed by whole body computed tomography scan (CT) and a CT of the sites of disease at baseline and repeated after 4-6 weeks of treatment, at 2-3 months, then every 3 months.

\subsubsection{Efficacy assessment}

All cases were centrally reviewed at INT. Response to treatment was assessed by Response Evaluation Criteria in Solid Tumour (RECIST), version 1.1, and by Choi criteria [17]. Choi criteria are based on changes in tumour size ( $>10 \%$ maximum diameter) and density following contrast administration on CT.

OS and PFS were estimated using the Kaplan-Meier method. Patients without evidence of progression and interrupting treatment with pazopanib for any reason were censored at the last tumour assessment. Patients alive or lost to follow-up were censored at the last contact.

\subsubsection{Morphology and immunophenotype}

Diagnosis was centrally reviewed according to the last World Health Organisation (WHO) classification [3]. All selected cases were positive for CD34 and STAT6. Two additional cases included in the namedbase programme were excluded from this study since SFT diagnosis was not confirmed by pathologic review.

\subsubsection{Role of the funding source}

Glaxo Smith Kline provided pazopanib on a case by case basis, and was informed of the results. The corresponding author had the final responsibility for the decision to submit the paper for publication, and wrote the manuscript with all the other authors. The Company played no role in writing or revising the manuscript.

\section{Results}

\subsection{Experimental model and pharmacological studies}

\subsubsection{Antitumour activity studies}

A significant tumour growth inhibition was observed following treatment with the different anti-angiogenic agents, even if at a different extent. The only exception was pazopanib that showed a negligible antitumour effect throughout the experiment (Fig. 1, Table 1). After the first 4 weeks of treatment, the antitumour effect was maximum for regorafenib and less pronounced for sorafenib, sunitinib, bevacizumab and axitinib. In all the treated animal groups, tumour growth was resumed following drug withdrawal, although different growth 


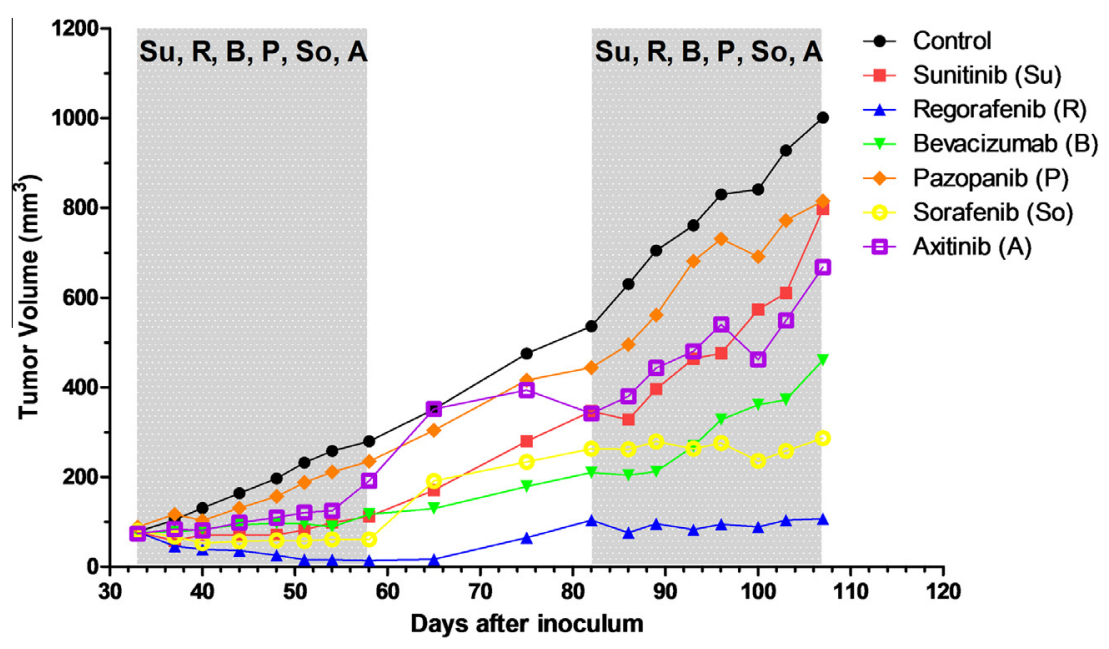

Fig. 1. Efficacy of pazopanib and other antiangionenic drugs against solitary fibrous tumour xenotransplanted into SCID mice. The treatment duration is indicated by the grey bar.

Table 1

Antitumour activity of pazopanib and other antiangiogenic drugs on solitary fibrous tumour xenograft.

\begin{tabular}{lllll}
\hline Drug & Dose $(\mathrm{mg} / \mathrm{kg})$ & Schedule & Route & Max TVI\% $(\mathrm{day})$ \\
\hline Pazopanib & 100 & $\mathrm{qd} \times 5 \mathrm{~d} / \mathrm{w} \times 4 \mathrm{w} \times 2$ & p.o & $21(86)$ \\
Sorafenib & 60 & $\mathrm{qd} \times 5 \mathrm{~d} / \mathrm{w} \times 4 \mathrm{w} \times 2$ & p.o & $78(58)^{* *}$ \\
Sunitinib & 40 & $\mathrm{qd} \times 5 \mathrm{~d} / \mathrm{w} \times 4 \mathrm{w} \times 2$ & p.o & $65(51)^{* *}$ \\
Regorafenib & 30 & $\mathrm{qd} \times 5 \mathrm{~d} / \mathrm{w} \times 4 \mathrm{w} \times 2$ & p.o & $95(65)^{* *}$ \\
Axitinib & 25 & $2 \mathrm{qd} \times 5 \mathrm{~d} / \mathrm{w} \times 4 \mathrm{w} \times 2$ & p.o & $51(54)^{*}$ \\
Bevacizumab & 4 & $\mathrm{q} 3-4 \mathrm{~d} / \mathrm{w} \times 4 \mathrm{w} \times 2$ & i.p & $70(89)^{* *}$ \\
\hline
\end{tabular}

${ }^{*} p<0.05$ and ${ }^{* *} p<0.01$ versus controls: Student's $t$ test; TVI: tumour volume inhibition.

kinetics in the rest period were observed for the different compounds. In the case of regorafenib and sorafenib, the second run (4 weeks) of treatment, delivered after a 3-week rest, stabilised tumour volume for the duration of treatment (Fig. 1).

No sign of toxicity was registered, with the exception of regorafenib that caused a $10 \%$ body weight loss during the second run of treatment.

\subsubsection{Pathologic evaluation of drug-treated xenograft}

Pathologic evaluation was carried-out on tumour samples obtained after $2 \mathrm{~h}$ from the last drug administration, at the end of the first and of the second run of treatment. No histological changes were observed between treated and untreated tumours.

\subsubsection{PDGFRB, VEGFR2 and ERK1/2 expression/ activation}

After the first 4 weeks of treatment sorafenib induced a decrease in PDGFRB expression/activation, regorafenib and especially axitinib induced a decrease in PDGFRB activation, while sunitinib, bevacizumab and pazopanib did not (Fig. 2A). Sunitinib, bevacizumab and regorafenib induced a weak decrease of VEGFR2 activation, while all the other treatments did not (Fig. 2B). The only drug that induced a decrease
A

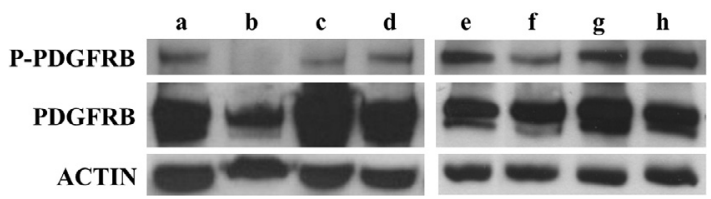

B

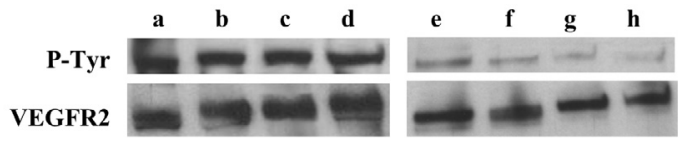

C

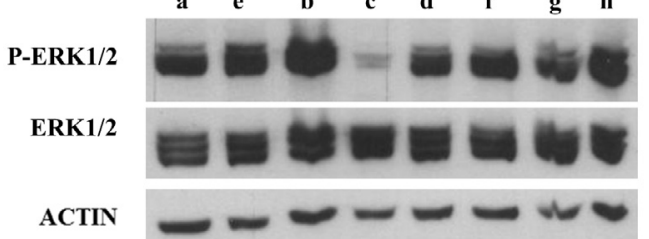

Fig. 2. Biochemical analyses of samples derived from mice treated with anti-angiogenic drugs. Panel A: Western blotting (WB) analysis of PDGFRB. Panel B: Immunoprecipitation/WB of VEGFR2. Panel C: WB analysis of ERK1/2. Mice were treated with sorafenib (b), axitinib (c), pazopanib (d), regorafenib (f), sunitinib (g), bevacizumab (h) for 4 weeks. No treated mice were used as control (a and e). The PPDGFRB, P-Tyr, P-ERK1/2 panels identify the phosphorylated proteins; PDGFRB, VEGFR2, ERK1/2 panels indicate the expression of the corresponding proteins. Anti-actin antibody was used to normalise the results. 
Table 2

Patient characteristics and clinical findings.

\begin{tabular}{|c|c|c|c|c|c|c|c|c|c|}
\hline $\begin{array}{l}\text { Patient } \\
\text { ID }\end{array}$ & Sex & $\begin{array}{l}\text { Primary } \\
\text { tumour site }\end{array}$ & $\begin{array}{c}\text { Prior } \\
\text { chemotherapy } \\
\text { (response) }\end{array}$ & $\begin{array}{l}\text { Age at time of } \\
\text { pazopanib } \\
\text { (years) }\end{array}$ & $\begin{array}{c}\text { Tumour type at } \\
\text { time of } \\
\text { pazopanib }\end{array}$ & $\begin{array}{c}\text { Disease } \\
\text { extension }\end{array}$ & $\begin{array}{c}\text { Best Response Evaluation } \\
\text { Criteria in Solid Tumour } \\
\text { (RECIST) response }\end{array}$ & $\begin{array}{c}\text { Best } \\
\text { Choi } \\
\text { response }\end{array}$ & $\begin{array}{l}\text { PFS } \\
(\mathrm{mos}\end{array}$ \\
\hline 1 & $\mathrm{~F}$ & Pelvis & Yes (NV) & 53 & MSFT & Abdomen, bone & SD & SD & 5 \\
\hline 2 & $\mathrm{~F}$ & Pleura & Yes (NV) & 73 & MSFT & $\begin{array}{l}\text { Thoraco-abdominal } \\
\text { wall, bone }\end{array}$ & SD & SD & 3 \\
\hline 3 & M & Pleura & $\begin{array}{l}\text { Yes (stable } \\
\text { disease) }\end{array}$ & 63 & DSFT & Thoracic wall, lung & PD & PD & 1 \\
\hline 5 & M & Pleura & Yes (no) & 75 & DSFT & Lung, soft tissue & PD & PD & 2 \\
\hline 6 & $\mathrm{~F}$ & Abdomen & Yes (no) & 77 & MSFT & Abdomen, lung & SD & PR & 15 \\
\hline 7 & M & Kidney & Yes (yes) & 33 & DSFT & $\begin{array}{l}\text { Lung, soft tissue, } \\
\text { bone }\end{array}$ & PD & PD & 2 \\
\hline
\end{tabular}

F: female; M: male; NV: not valuable; MSFT: malignant solitary fibrous tumour; DSFT: dedifferentiated solitary fibrous tumour; SD: stable disease; PD: progressive disease; PR: partial response; PFS: progression free survival; mos: months.

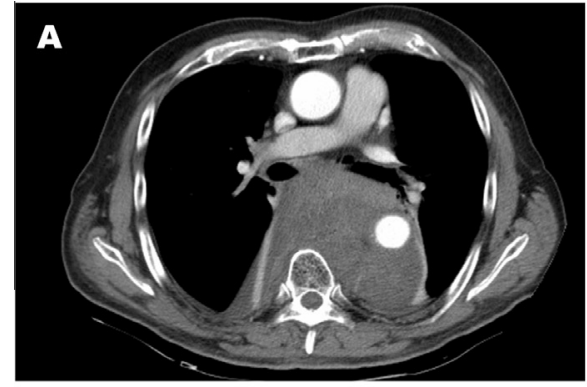

baseline

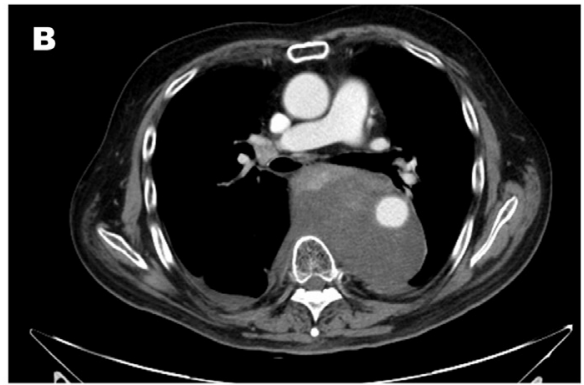

+3 months

\section{pazopanib $800 \mathrm{mg} / \mathrm{day}$}

Fig. 3. Response to pazopanib. Computed tomography (CT) scan (arterial phase after contrast medium). Panel A shows a intrathoracic lesion from pleural solitary fibrous tumour at baseline. Three months after starting pazopanib a response was observed, marked by a decrease in tumour size and pleural effusion, and a decrease in tumour density (Panel B).

in ERK1/2 activation after one cycle of treatment was axitinib (Fig. 2C, line c) but this result was not confirmed by the analysis performed on the tumour samples excised after the second run (data not shown).

\subsection{Patients}

Six patients received pazopanib from May 2012 to October 2013. All cases completed their treatment (four progression; two toxicity) and were evaluable for response.

\subsubsection{Patients}

Table 2 summarises clinical findings.

Mean age was 62 years. PS was $\leqslant 2$ in all cases. All patients had progressed before starting treatment. Morphology was consistent with a diagnosis of malignant-SFT in three patients and dedifferentiated-SFT in the other three.

Median treatment duration was 3 months (range: $1-$ 15). All patients started pazopanib $800 \mathrm{mg} /$ day. Two patients interrupted definitively their treatment due to toxicity (G3 liver toxicity: 1; G3 cardiac toxicity: 1). Toxicity resolved upon discontinuation.

\subsubsection{Response}

Best RECIST responses were three stable disease (SD) and three progressive disease (PD). According to Choi criteria best response was partial response (PR) in one, confirmed at 3 months, SD in two and PD in three (Fig. 3).

At a median 10-month follow-up, three patients are alive, three dead. Median PFS by RECIST was 3 months (range 1-15).

In one patient progressive under pazopanib, sunitinib $37.5 \mathrm{mg}$ /day was started with response after 3 months (Fig. 4).

\section{Discussion}

In a mouse model of dedifferentiated-SFT, pazopanib showed the lowest antitumour activity (21\%TVI), when 


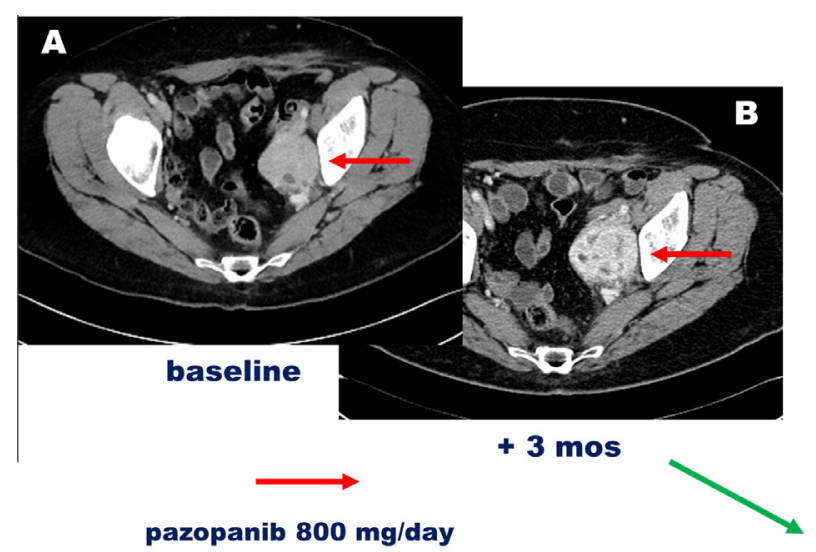

sunitinib $37.5 \mathrm{mg} / \mathrm{day}$

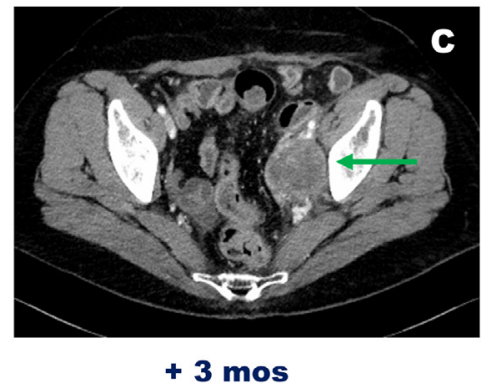

Fig. 4. Response to sunitinib after failure to pazopanib. Computed tomography (CT) scan (arterial phase after contrast medium). Panel A shows a pelvic lesion from pelvic solitary fibrous tumour at baseline. Three months after starting pazopanib a progression was observed, marked by an increase in tumour size and contrast uptake was observed (Panel B). On this basis pazopanib was interrupted and sunitinib was started with evidence of response 3 months later in terms of minor tumour shrinkage and hypodensity (Panel C).

compared to axitinib, bevacizumab, regorafenib, sorafenib and sunitinib, while regorafenib was the most active compound ( $95 \%$ TVI). Sorafenib, bevacizumab and sunitinib were also markedly active $(78 \%, 70 \%$ and $65 \%$ TVI, respectively), whereas axitinib was marginally active $(51 \% \mathrm{TVI})$. In a series of six patients with progressing metastatic SFT treated with pazopanib, the best responses according to RECIST were three SD and three $\mathrm{PD}$, corresponding to one PR, two SD, three PD by Choi criteria, with a 3-month median PFS. All patients showing a tumour disease stabilisation under pazopanib were malignant-SFT, while those who progressed were highgrade dedifferentiated-SFT. Interestingly a response was subsequently obtained administering sunitinib in a patient who did not respond to pazopanib.

SFT represents a very rare disease and very few data are available on its sensitivity to pazopanib, that is the only antiangiogenic agent approved for STS treatment. Our preclinical results showed that pazopanib is less active compared to other antiangiogenics in a high-grade dedifferentiated-SFT mouse model [10]. By contrast regorafenib was found to be the most interesting compound. All the tested antiangiogenics showed a cytostatic effect. In all cases, indeed, tumour re-growth was observed after treatment discontinuation. This is consistent with the evidence that tumour samples after treatment with the different agents were made by viable cells in all cases. Of interest, among RTK inhibitors, pazopanib and axitinib were found to be less active than sunitinib, sorafenib and regorafenib. In particular, pazopanib could obtain only a very modest decrease in tumour growth compared to control. A clear explanation for that is not yet available. The larger spectrum of kinase inhibition that marks sunitinib and especially sorafenib and regorafenib compared to pazopanib could make the difference. This hypothesis is corroborated by the evidence that, among the different antiangiogenics, only treatment reiteration (after a 3-week rest) with sorafenib and regorafenib, i.e. the two drugs with the largest spectrum of targets, was able to stabilise tumour volume. Biochemical analysis of PDGFRB, VEGFR2 and ERK1/2 after treatment showed that pazopanib did not switch-off any of the targets, consistently with its marginal activity. In contrast to what we expected, a decrease in kinase activation was not detected even in tumour samples which have responded to treatment. The presence of viable tumour cells in post-treatment samples even in tumours that significantly shrank strongly suggests the presence of resistant cells. We lack a definitive explanation for this observation and new experiments are in place to further investigate the different sensitivity of our SFT model to the antiangiogenics. We recently found that the antitumour effect of sunitinib in SFT patients is ascribable, at least in part, to antiangiogenic drug immunomodulating functions [18]. However, it is difficult to envisage such an effect in our patient-derived xenografts growing into immunodeficient hosts, such as SCID mice. To be noticed, the inhibitory effect of the different antiangiogenics on their target proteins is characterised by a different kinetics. As a consequence, the evaluation 
of kinase phosphorylation status at a single time point, as we made in our experiment, could have prevented the possibility to compare properly the biochemical effects of the different compounds. We have started new experiments in tumour samples obtained at different intervals from mice treated with the different agents aimed at comparatively evaluating changes in the transcriptome as well as in the phosphorylation status of proteins belonging to relevant pathways.

The French group already reported on two SD out of six SFT patients treated with pazopanib (PFS 8 and 14 months, respectively) [9]. Our clinical data, even if retrospective and on a small number of patients, confirm that pazopanib had a modest activity in this sarcoma subtype and, interestingly, are in line with the preclinical results. No RECIST responses could be observed but in one patient the effect of pazopanib was marked by a minor $(<30 \%)$ decrease in tumour size and by a decrease in tumour density, thus classifying for a PR by Choi criteria [11]. As already described for SFT treated with bevacizumab, sunitinib and sorafenib, Choi criteria [4-9], originally conceived for GIST receiving imatinib [11], differed from RECIST in assessing response to therapy. Of note, the patient with a Choi response had the best PFS (15 months). Our results seem to suggest a lower level of activity of pazopanib in SFT compared to sunitinib and bevacizumab plus temozolomide $[5,6]$. Median PFS of patients treated with pazopanib was 3 months, while a retrospective study on sunitinib in 31 SFT patients showed a Choi RR of about $50 \%$ with a 6-month median PFS [5]. Again, a Choi RR of $79 \%$ with a median PFS of 9.7 months was reported in SFT patients treated with bevacizumab and temozolomide [6]. Interestingly, patients who progressed under pazopanib had dedifferentiated-SFT, while the responsive case carried a malignant-SFT. This observation suggests that pazopanib might be more active in less aggressive cases, as already observed in SFT patients treated with sunitinib, and by contrast to what was observed with cytotoxic chemotherapy [19]. Worth noting, we obtained a response to sunitinib in a patient progressive to pazopanib consistently with the preclinical evidence of a non-superimposable activity of antiangiogenics in SFT.

Our results need to be confirmed prospectively. A European study on pazopanib in advanced SFT aimed at evaluating Choi response as primary end-point has just started. In addition, based on preclinical data showing that regorafenib is the most promising antiangiogenic agent, a prospective study on regorafenib in SFT is actually under discussion.

\section{Funding}

Associazione Italiana per la Ricerca sul Cancro (AIRC) grants 14102 to S. Pilotti and 11817 to T. Negri.

\section{Disclosures}

S. Stacchiotti, S. Provenzano, E. Fumagalli received research funds from Glaxo Smith Kline, Pfizer, Bayer.

A.P. Dei Tos and PG Casali received honoraria, compensation for advisory boards, research funds and travel coverage from Glaxo Smith Kline, Pfizer, Bayer.

G. Grignani received travel coverage by Glaxo.

A. Gronchi received honoraria from Pfizer.

\section{Conflict of interest statement}

None declared.

\section{References}

[1] Schutz FA, Choueiri TK, Sternberg CN. Pazopanib: clinical development of a potent anti-angiogenic drug. Crit Rev Oncol Hematol 2011;77:163-71.

[2] Van der Graaf WT, Blay JY, Chawla SP, et al. Pazopanib for metastatic soft-tissue sarcoma (PALETTE): a randomised, double-blind, placebo-controlled phase 3 trial; EORTC Soft Tissue and Bone Sarcoma Group; PALETTE study group. Lancet 2012;379:1879-86.

[3] Fletcher CDM et al., editors. World Health Organization (WHO) classification of tumours of soft tissue and bone. Pathology and genetics. Lyon: IARC Press; 2013, pp. 80-82.

[4] Domont J, Massard C, Lassau N, et al. Hemangiopericytoma and antiangiogenic therapy: clinical benefit of antiangiogenic therapy (sorafenib and sunitinib) in relapsed malignant haemangioperyctoma/solitary fibrous tumour. Invest New Drugs 2010;28:199-202.

[5] Stacchiotti S, Negri T, Libertini M, et al. Sunitinib malate in solitary fibrous tumor. Ann Oncol 2012;23:3171-9.

[6] Park MS, Patel SR, Ludwig JA, et al. Activity of temozolomide and bevacizumab in the treatment of locally advanced, recurrent, and metastatic hemangiopericytoma and malignant solitary fibrous tumor. Cancer 2011;117:4939-47.

[7] Valentin T, Fournier C, Penel N, et al. Sorafenib in patients with progressive malignant solitary fibrous tumors: a subgroup analysis from a phase II study of the French Sarcoma Group (GSF) GETO). Invest New Drugs 2013;31:1626-7.

[8] Constantinidou A, Jones RL, Olmos D, et al. Conventional anthracycline-based chemotherapy has limited efficacy in solitary fibrous tumour. Acta Oncol. 2012;51:550-4.

[9] Levard A, Derbel O, Méeus P, et al. Outcome of patients with advanced solitary fibrous tumors: the Centre Léon Bérard experience. BMC Cancer. 2013;13:109.

[10] Stacchiotti S, Tortoreto M, Bozzi F, et al. Dacarbazine in solitary fibrous tumor: a case series analysis and preclinical evidence vis-àvis temozolomide and antiangiogenics. Clinical Cancer Res 2013;19:5192-201.

[11] Robinson DR, Wu YM, Kalyana-Sundaram S, et al. Identification of recurrent NAB2-STAT6 gene fusions in solitary fibrous tumor by integrative sequencing. Nat Genet 2013;45:180-5.

[12] Podar K, Tonon G, Sattler M, et al. The small-molecule VEGF receptor inhibitor pazopanib (GW786034B) targets both tumor and endothelial cells in multiple myeloma. Proc Natl Acad Sci U S A 2006;103:19478-83.

[13] Cassinelli G, Lanzi C, Tortoreto M, et al. Antitumor efficacy of the heparanase inhibitor SST0001 alone and in combination with antiangiogenic agents in the treatment of human pediatric sarcoma models. Biochem Pharmacol 2013;85:1424-32.

[14] Canu B, Fioravanti A, Orlandi P, et al. Irinotecan synergistically enhances the antiproliferative and proapoptotic effects of axitinib 
in vitro and improves its anticancer activity in vivo. Neoplasia 2011;13:217-29.

[15] Wang X, Zhang L, Goldberg SN, Bhasin M, Brown V, Alsop DC, et al. High-dose intermittent sorafenib shows improved efficacy over conventional continuous dose in renal cell carcinoma. J Transl Med 2011;9:220.

[16] Wilhelm SM, Dumas J, Adnane L, Lynch M, Carter CA, Schütz G, et al. Regorafenib (BAY 73-4506): a new oral multikinase inhibitor of angiogenic, stromal and oncogenic receptor tyrosine kinases with potent preclinical antitumor activity. Int J Cancer. 2011;129:245-55.

[17] Choi H, Charnsangavej C, Faria SC, et al. Correlation of computed tomography and positron emission tomography in patients with metastatic gastrointestinal stromal tumor treated at a single institution with imatinib mesylate: proposal of new computed tomography response criteria. J Clin Oncol 2007:25:1753-9.

[18] Tazzari M, Negri T, Rini F, et al. Adaptive immune contexture at the tumour site and downmodulation of circulating myeloidderived suppressor cells in the response of solitary fibrous tumour patients to anti-angiogenic therapy. Br J Cancer 2014. http:// dx.doi.org/10.1038/bjc.2014.437.

[19] Stacchiotti S, Libertini M, Negri T, et al. Response to chemotherapy of solitary fibrous tumor: a retrospective study. Eur J Cancer 2013;49:2376-83. 